\title{
Research on Transmission System of Video Monitoring for theater Based on IP Multicast
}

\author{
Zhen $\mathrm{Li}^{1,2,3}$, Liu Gao ${ }^{1,2,3}$ and Hui Ren ${ }^{1,2,3}$ \\ ${ }^{1}$ School of Information and Engineering, Communication University of China \\ ${ }^{2}$ Key Laboratory of Acoustic Visual Technology and Intelligent Control System, Ministry of Culture \\ ${ }^{3}$ Beijing Key Laboratory of Modern Entertainment Technology \\ Beijing, China \\ E-mail: lizhen@cuc.edu.cn
}

\begin{abstract}
Keywords: IP multicast, theater monitoring video, video transmission, Winsock
Abstract. At present, with the video data growth, in the transmission system of theater video monitoring, it uses the communication way of IP unicast and IP broadcast to transmit video data, which leads to the rapid consumption of bandwidth. In order to solve the above problem, this paper proposes to adopt the IP multicast for transmitting video data in the transmission system of theater video monitoring. Particularly, this paper introduces the IP multicast technology, and the design concept that using Winsock interface to set up the transmission system of theater video monitoring.
\end{abstract}

\section{Introduction}

In recent years, with the rapid development of theater, the application of the transmission system of theater video monitoring has become more and more popular. At present, the system consistes of multiple network cameras and monitoring terminals with the communication way of IP unicast and IP broadcast, which lead to the rapid consumption of bandwidth, and network congestion problem. Compared with the IP unicast and IP broadcast, IP multicast uses the communication way of one point to multipoint or multipoint to multipoint. So if the transmission system of theater video monitoring uses the IP multicast communication, it will save bandwidth and server payload along with increasing users. This paper proposes the method that using Winsock interface based on IP multicast to set up the transmission system of theater video monitoring, which can improve the video transmission efficiency.

\section{IP Multicast}

IP Multicast Communication. The multicast group consists of the hosts using the same multicast address, which is the range of 224.0.0.0 239.255.255.255 of IP address. The member of multicast group can change at any time. In addition, a host can also send data to one multicast group, which doesn't belong to this multicast group [1].

IP multicast uses the multicast distribution tree to describe the data transmission path in the network. There are two kinds of multicast distribution tree, including the source-based tree and shared tree. Source-based tree lies in the shortest path routing protocol, so also known as the shortest path tree (SPT). Shared tree sends data from the multicast source to the public multicast router, then to the hosts of multicast group [2].

In order to realize IP multicast communication, firstly, hosts configure the hardware interface for identifying the IP multicast address. Secondly, the multicast router queries the member of multicast group periodically to estimate whether or not they are still involved. As long as there is a host, router will continue to receive data. Finally if all hosts leave the multicast group, router will receive the "leave message" of IGMP, then the router will immediately query if the members is still involved. If the multicast group are still active members, router will continue to transmit data, otherwise it will stop doing it [3].

IP Multicast Protocol. There are two kinds of multicast protocol, including the group membership protocol and the multicast router protocol. 
IGMP connecting group membership to routers directly is a group membership protocol, which is responsible for the information establishment and maintenance of group membership.

According to the distribution information of group members, there are two kinds of multicast router protocol, including PIM-SM (Protocol Independent Multicast-Spare Mode) and PIM-DM (Protocol Independent Multicast-Dense Mode). PIM-DM assumes that the members of group are densely distributed in the network, and there is enough bandwidth in the network. And it pushes the data to the multicast router through the broadcasting technology. While PIM-SM assumes that the members of group are sparsely distributed in the network $[4,5]$.

Implementation of IP Multicast. Winsock provides a series of application programming interface to achieve the IP multicast communication. In the IP multicast communication, the receiving end joins in a multicast group, then the sending end sends data to the corresponding multicast address. The flow chart of Realizing IP multicast using Winsock2 refers to Fig. 1.

Sending end

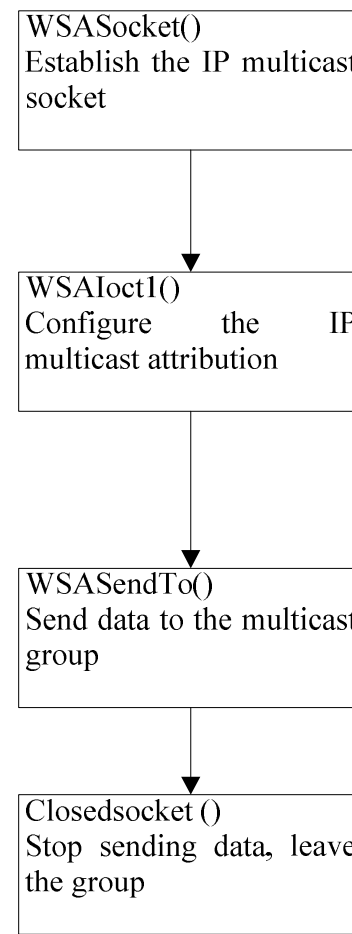

Receiving end

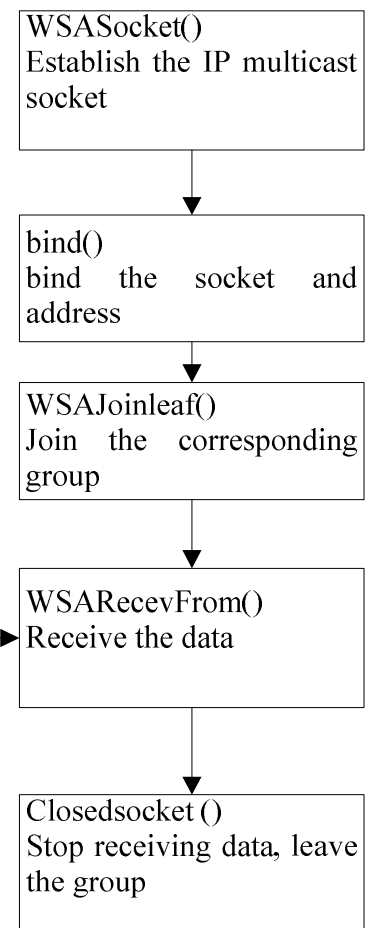

Fig. 1 The flow chart of IP multicast communication

\section{Application of the Transmission System of Theater Video Monitoring on IP Multicast}

The Overall Framework of System. The system adopts the C/S structure based on Socket, as is shown in Fig. 2. It mainly consists of the video data process module, video data control module, video data sending module, and video data receiving module.

(1) Video data process module.

The module mainly realizes the function of collection, extraction and dynamic storage of the real-time video data through the SDK kit provided by the camera manufacturer.

(2) Video data control module.

The module mainly realizes the function of switching video data to transmit according to the partial demand.

(3) Video data sending module.

The module is responsible for sending real-time video data to a number of remote monitor terminals in IP multicast communication.

(4) Video data receiving module. 
The module is responsible for receiving real-time video data from the video server in IP multicast communication.

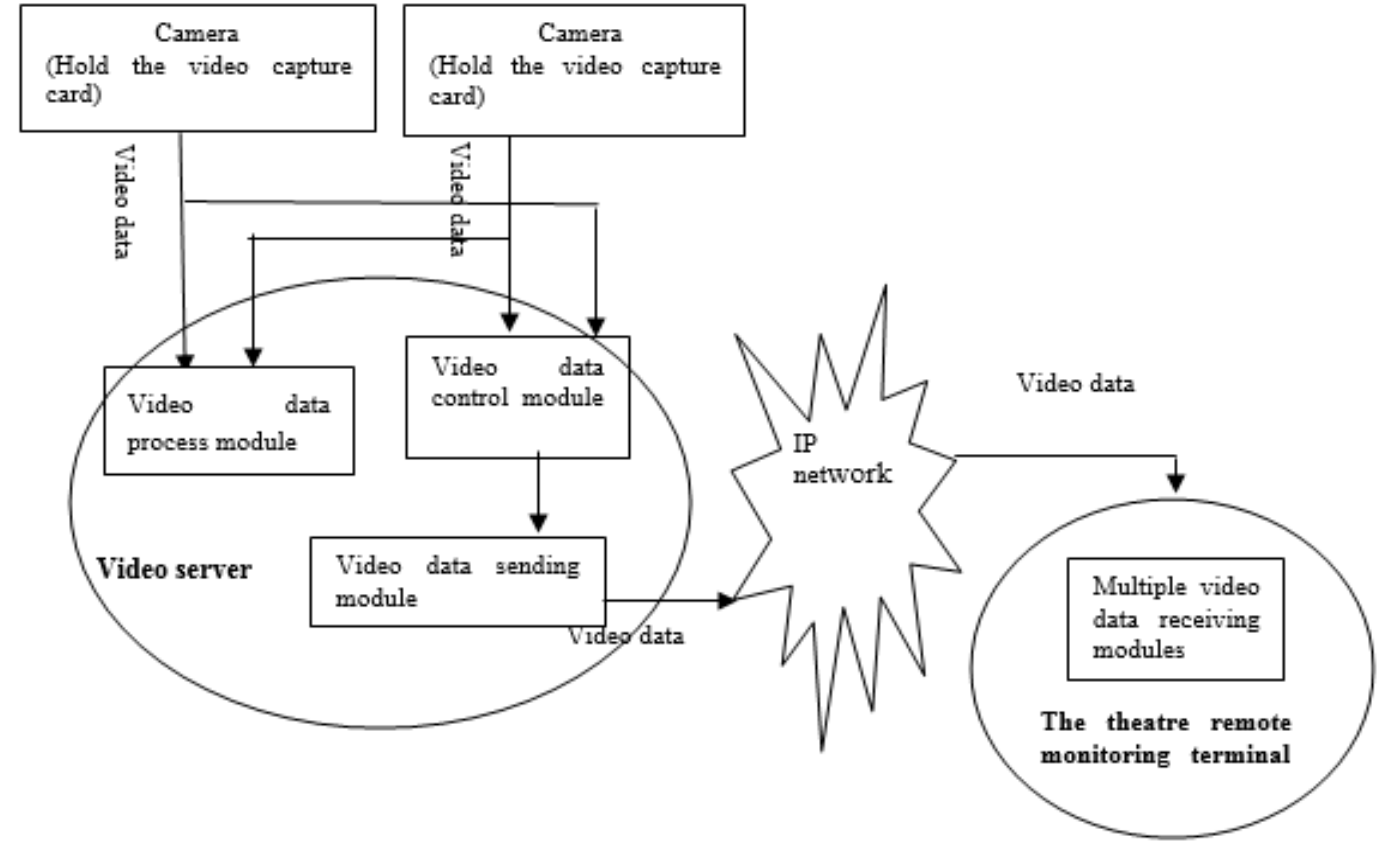

Fig. 2The overall framework figure of system

Design of Video Sending Module. This module is applied for the video server. The main task is to send video data in the transmission system of theater video monitoring. The module need to solve the main problem about sending video data in real-time, convenient, and efficient. This paper uses the Winsock interface to design the video sending module based on UDP protocol. The flow chart of sending the real-time video data refers to Fig. 3.

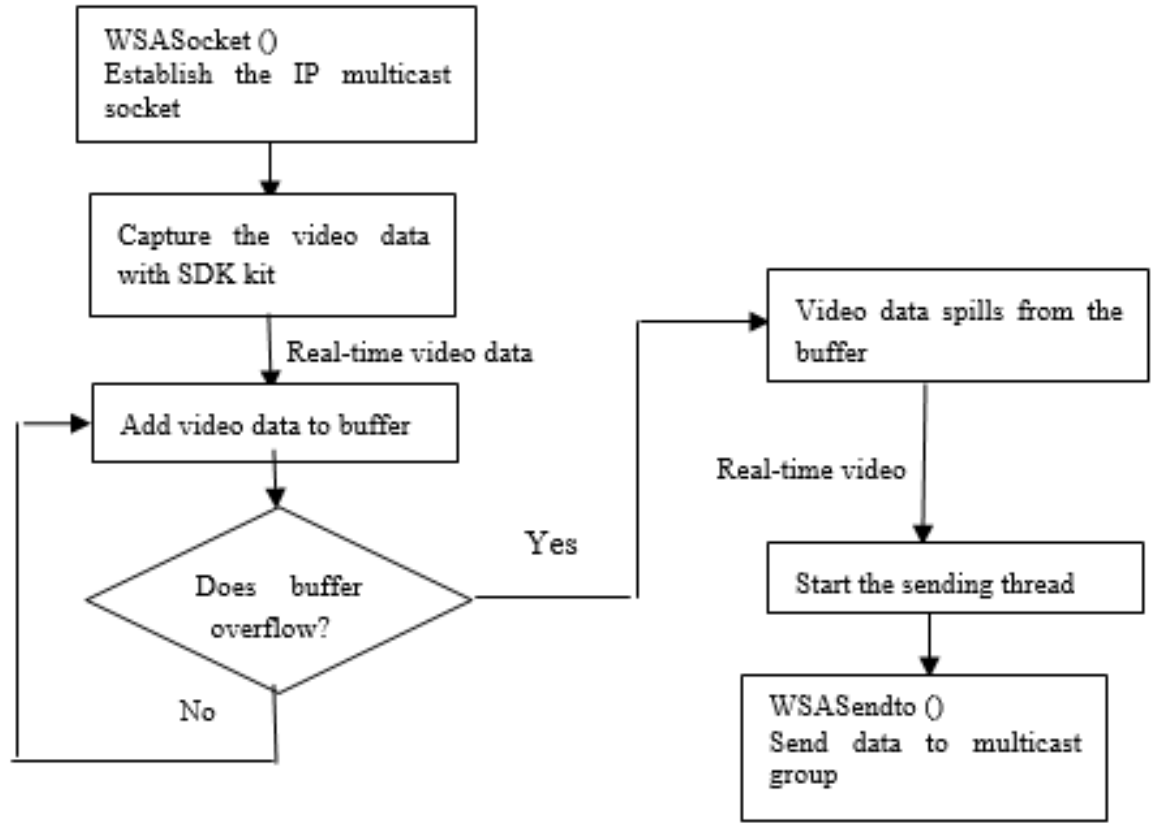

Fig. 3The flow chart of sending video data

Now, there are two ways of data sending, including the file-transmission and real-time transmission. The paper uses the real-time transmission way. The real-time video data comes from the camera with the video capture and compression card. In the process of sending the real-time video, through the SDK kit provided by the camera manufacturer, it collects the video data. In addition, the video sending module should set the buffer to store the acquired data so that the remote monitoring terminal can swatch video smoothly. 
Design of Video Receiving Module. This module is applied for the theater remote monitoring terminal. The main task is to receive video data from the video sending module. This paper uses the Winsock interface to design the video receiving module based on UDP protocol. The flow chart of receiving the real-time video data refers to Fig. 4.

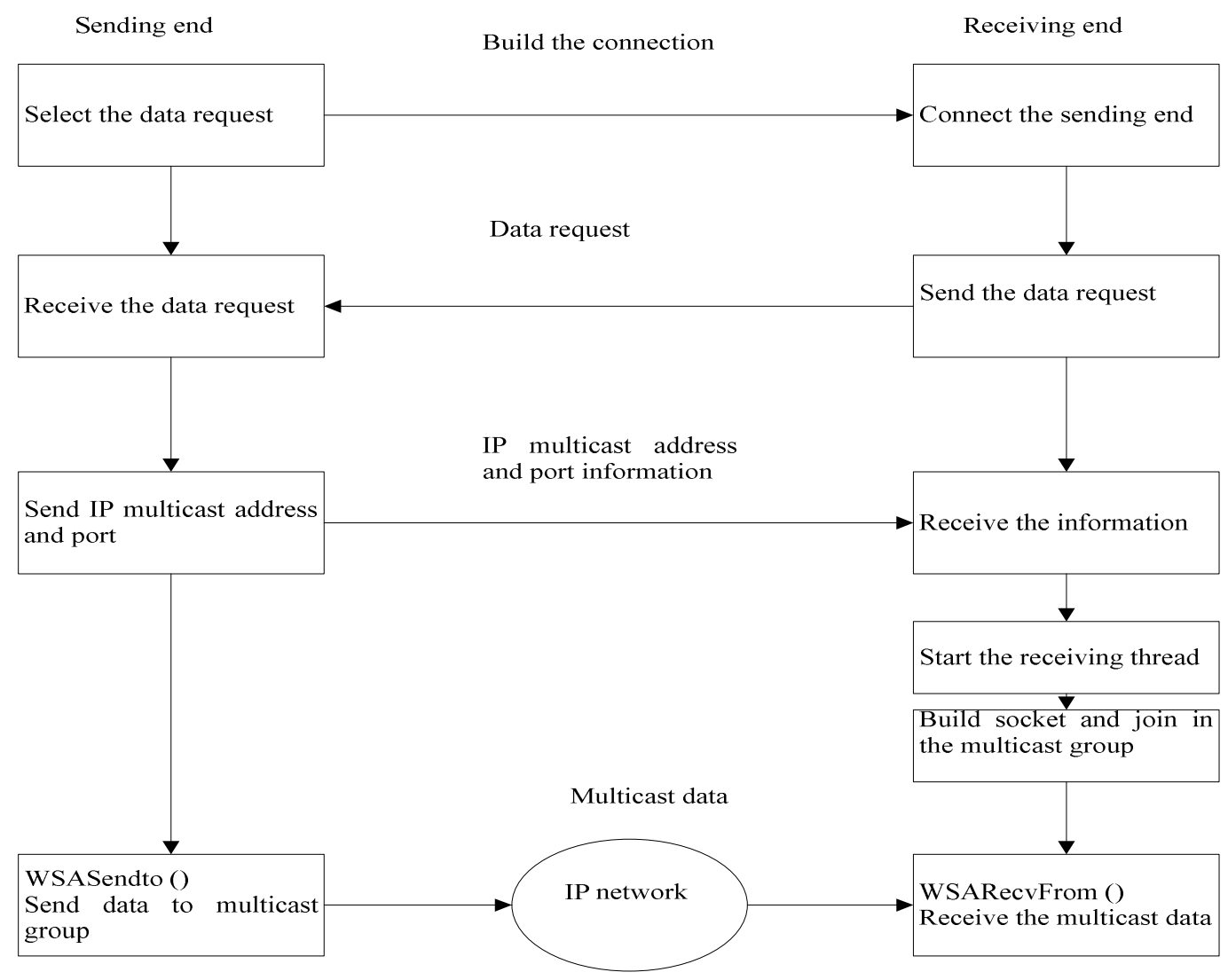

Fig. 4The flow chart of receiving video data

\section{Summary}

This paper mainly discusses the IP multicast and its application for the transmission system of theater video monitoring, which will realize the multiple transmission and real-time playback of video data. The proposed system can not only save the network bandwidth, but also improve the video data transmission efficiency by using Winsock interface based on IP multicast. With the urgent demand for high bandwidth communication, IP multicast application will have broad prospect.

\section{Acknowledgment}

This paper was financially supported by the national science and technology planning project "Study and application demonstration on the key technology of stage effect" (Item Number: 2012BAH38F00)

\section{References}

[1] Guo Hao P. (2007). Research and implementation of video transmission system based on IP multicast [J]. Computer and Modernization 142(6):81-84.

[2] Yong Cheng Y, Yuan Zhi Y, Gui Ming H. (2003). IP multicast and its application based on Winsock [J]. Computer Application 23(6):247-250. 
[3] Dong Xing J. Windows Sockets Network programming [M]. Beijing: Tsinghua University Press, 1999.

[4] Xiao Lian C. (2003) Research of the digital video transmitting system based on IP multicast [D]. Nanjing: 01-68.

[5] P.G. Clem, M. Rodriguez, J.A. Voigt and C.S. Ashley, U.S. Patent 6,231,666. (2001) 